\title{
LOS ELEMENTOS BÁSICOS PARA LA FORMULACIÓN DE POLÍTICAS CIENTÍFICAS, TECNOLÓGICAS Y DE INNOVACIÓN PARA LA COHESIÓN SOCIAL. UNA VISIÓN CTS ${ }^{1}$
}

\section{THE FOUNDATIONS OF SCIENTIFIC, TECHNOLOGICAL AND INNOVATION POLICY PROPOSALS FOR SOCIAL COHESION. A CTS POINT OF VIEW}

\author{
Ronny J. Viales Hurtado*
}

\begin{abstract}
RESUMEN
En este artículo se discuten algunos elementos básicos para la formulación de políticas científicas, en general, para la cohesión social, desde una perspectiva de Ciencia, Tecnología y Sociedad (CTS). Se toma en cuenta la necesidad de participación de actores estratégicos y de actores emergentes, los cuales deben integrar tanto a las autoridades políticas y científicas tradicionales como a la sociedad civil; así como, el giro deliberativo que puede coadyuvar a superar el déficit democrático en materia de políticas científicas. Se pondrá énfasis en los elementos de contexto y en la fase de percepción y definición del problema de política pública, según el planteamiento de Joan Subirats y se presentará una propuesta inicial para la participación en la formulación de políticas científicas.
\end{abstract}

PALABRAS CLAVE: POLÍTICA CIENTÍFICA * SOCIEDAD * CIENCIA * INVESTIGACIÓN * PARTICIPACIÓN SOCIAL

\section{ABSTRACT}

In this article, we discuss some basic foundations for the production of research policies for social cohesion from a STS perspective. It's taking into consideration the need of strategic and emergent actors' participation which includes political and scientist traditional authorities, and civil society. In addition we present the deliberative turn that helps to overcome the democratic deficit in scientific policies. Emphasis will be on the contextual factors and the phases of perception and definition of public policies, according to Joan

$1 \quad$ Este artículo es resultado parcial del Proyecto de Investigación "Los actores, la percepción y la definición el problema de las políticas científicas en América Central, en el contexto global, y su relación con la cohesión social. 1980-2014. Un análisis histórico desde la perspectiva CTS" (806-B6-191) inscrito en el Programa de Investigación "Ambiente, Ciencia, Tecnología y Sociedad (ACTS) Intersección entre Historia Ambiental y Estudios Sociales de la Ciencia, la Tecnología y la Sociedad (CTS)" (806-B6-901) del Centro de Investigaciones Históricas de América Central de la Universidad de Costa Rica.

* $\quad$ Centro de Investigaciones Históricas de América Central, Universidad de Costa Rica (CIHAC-UCR). ronny.viales@ucr.ac.cr 
Subirats's approach and we introduce an initial propose for the participation on the formulation of scientific policies.

KEYWORDS: SCIENTIFIC POLICIES $*$ SOCIETY $*$ SCIENCE * RESEARCH $*$ SOCIAL PARTICIPATION

\section{INTRODUCCIÓN}

El contexto de la "sociedad red" (Castells, 2005) y de la "sociedad tecnológica" (Queraltó, 2003) permite repensar las relaciones entre ciencia, tecnología, innovación y cohesión social desde los países periféricos, que evidencian una brecha tecnocientífica, provocada por una nueva división internacional del trabajo científico en el mundo, donde regiones como América Latina se debaten entre su inserción como integrados o dependientes (Kreimer, 2006).

Pero existe también una brecha social, que no ha permitido vincular los elementos anteriores, con miras a potenciar una mayor cohesión social, la cual disminuya las desigualdades sociales, que implica, desde nuestra perspectiva (Uso de la Ciencia para la Cohesión Social - UCICos, 2008), investigar ¿qué capacidades y estrategias facilitan o impiden a los actores sociales participar e influir en las discusiones de políticas científicas y tecnológicas? Asimismo, proponer mecanismos para disminuir la exclusión y la discriminación, así como aumentar la cohesión social e identificar instituciones, políticas, tecnologías y fuerzas-actores sociales que generan desigualdades (Wetmore, 2007).

En el año 2000, en el marco de la "Comisión de las Comunidades Europeas" (CEC por sus siglas en inglés), se inició un debate destacado sobre la relación entre "Ciencia, Sociedad y los ciudadanos en Europa", lo cual tenía dos aristas principales: por una parte, ¿cómo implementar políticas de investigación vinculadas con los intereses reales de la sociedad? y aunado con lo anterior, ¿cómo involucrar a la sociedad en la construcción de la agenda de investigación?, pregunta sobre la que se había hecho un abordaje anteriormente (Viales, 2010, pp.21-40). Para responder estas interrogantes, el telón de fondo estuvo constituido por la necesidad de indagar el conocimiento público de la ciencia, de incrementar el interés de las personas jóvenes para desarrollar carreras científicas, así como de ampliar el papel y el lugar de las mujeres en el campo de la investigación científica (CEC, 2000).

Para comprender las demandas sociales de investigación científica y de producción de conocimiento, es necesario que haya una interacción entre las ciencias, las humanidades y las ciencias sociales, lo que ayudará a una mejor aproximación al problema del desarrollo científico y tecnológico, favoreciendo el diálogo entre ciencia y sociedad, así como en el manejo del riesgo, en el sentido de que la ciencia $y$ la tecnología pueden contribuir a desarrollar un principio de vigilancia expectante, de precaución, debido a que las personas y los mass media evidencian que estas actividades pueden generar riesgos sociales debido a la incertidumbre científica. Por esta razón es importante la construcción de sistemas regulatorios de la actividad tecnocientífica que generen confianza (Harmon, Laurie y Haddow, 2013).

El planteamiento de Michael Gibbons et ál. (1994), al distinguir una nueva forma de producción del conocimiento, que denominan Modo 2 (que se produce en un contexto de aplicación; es transdisciplinario, heterogéneo $y$ se da en formas de organización diversas; es responsable socialmente $y$ reflexivo, al incorporar valores e intereses de otros grupos); permite el control de calidad (dimensiones cognitivas sociales, económicas, ambientales $y$ políticas) (Casas, 2004) que se contrapone, pero que coexiste, con el Modo 1 (en el que los problemas de investigación son definidos en el ámbito académico; conocimiento disciplinario; organización regida por las normas de la ciencia; no es responsable socialmente; transmitido por medio de formas de publicación académica; validado y evaluado por la comunidad de especialistas) (Casas, 2004), es decir, el denominado "modelo lineal" ha llevado, según Sheila Jasanoff, a la tesis de que 
los objetivos de la ciencia hoy son más dispersos, dependientes del contexto y orientados a los problemas científicos, por lo que estos cambios hacen necesarias nuevas formas de justificación pública de la investigación y del desarrollo científico (Jasanoff, 2003, p.226).

En el caso de América Latina, es importante sistematizar, desde una perspectiva CTS, es decir, desde el planteamiento de que la producción/construcción de conocimiento científico es una actividad intrínsecamente social (Sismondo, 2010), una visión sobre los modos de participación y el impacto en las distintas fases del proceso de elaboración de las políticas $y$ agendas de CTI que incluya:

$\diamond \quad$ La producción de conocimiento e información sobre los problemas científicos: toma de conciencia de los problemas por parte de la opinión pública, los actores implicados y el sistema político.

$\diamond \quad$ El inicio de debates sociales, la formación de actitudes y de opiniones sobre los problemas científicos.

$\diamond \quad$ La discusión sobre los valores y la ética de la formulación de las políticas científicas para la cohesión social.

$\diamond \quad$ El acotamiento y la selección de alternativas, la definición de la agenda y la estructuración del debate político sobre estas opciones.

$\diamond \quad$ La efectividad del proceso por el cual se llega a la toma de decisiones.

$\diamond \quad$ La rendición de cuentas sobre estas decisiones.

En la región han persistido limitantes para el desarrollo tecnocientífico, que han coexistido con imaginarios sobre el papel que la ciencia y la tecnología pueden jugar en el logro de sociedades más igualitarias (Vessuri, 2003). Ahora se abre la posibilidad de crear nuevos espacios para negociar las cuestiones que pueden formar parte de la agenda del desarrollo Científico, Tecnológico y de Innovación (CTI) de la región latinoamericana, a partir de metodologías participativas para integrar a los diferentes actores en la definición y la gestión de estas políticas a partir de sus necesidades. A continuación se problematiza este nuevo contexto.

\section{¿QUÉ ES LA COHESIÓN SOCIAL Y CUÁL ES SU} RELACIÓN CON LAS POLÍTICAS DE CTI?

La idea de la cohesión social ha sido un tema de discusión clásico en el ámbito de las ciencias sociales $y$ esta se ha comprendido como el grado de acuerdo entre los miembros de un grupo social, además de la percepción de pertenecer a una sociedad o a un proyecto común. En otras palabras, la cohesión social permite aproximarse al porqué las sociedades permanecen unidas (Casas, 2012, p.563). Es importante tomar en consideración, la perspectiva que ha sido impulsada por la Comisión Económica para América Latina (CEPAL), en el contexto de la desigualdad creciente de este subcontinente latinoamericano, que se puede sintetizar mediante la relación entre dos dimensiones: una material, objetiva, que se vincula con los mecanismos de inclusión en las sociedades (principalmente el trabajo, la educación, los derechos y la distribución de la riqueza), a la que habría que incluir la стI; $y$ una subjetiva, que se vincula con los valores y los comportamientos de los individuos en la sociedad (tales como la confianza, la solidaridad $y$ el sentimiento de pertenencia, entre otros) (CEPAL, 2007).

Dichos componentes son construidos históricamente $y$ pueden entrar en crisis cuando las ideas centrales sobre las que se fundamenta una sociedad empiezan a erosionarse. De ahí que la cohesión social dependa estrechamente de logros científicos, económicos, sociales, políticos y culturales que se manifiesten en niveles aceptables de equidad social, de la percepción de la equidad ante la justicia, del acceso al trabajo en condiciones no precarias, del acceso a la educación como ámbito que posibilita la movilidad social, de la igualdad en términos de derechos ciudadanos $y$ del reconocimiento genuino de las diversas culturas e identidades (Szmukler, 2008).

Como ha planteado Hernán Thomas, en general, la tecnología es un tema poco considerado en las ciencias sociales $y$ en particular, en los estudios sobre pobreza y marginalidad; no 
obstante, cuando se aborda el tema, se utilizan perspectivas deterministas lineales: o consideran que la tecnología determina el cambio social (determinismo tecnológico) o que la sociedad determina la tecnología (determinismo social), lo cual trae como resultado una división entre problemas sociales y problemas tecnológicos. Este autor propone desarrollar una visión socio-técnica para superar la dicotomía anterior, partiendo del hecho de que "las sociedades son tecnológicamente construidas al mismo tiempo que las tecnologías son socialmente configuradas" (Thomas, 2011).

Por lo anterior, cuando se formulan políticas científicas, antes que partir de determinismos lineales, se debería comprender, desde un enfoque constructivista, que la tecnología es un agente de cambio social. El propio Thomas (2011) ha señalado que esto es así porque las tecnologías: plantean condicionantes para los actores y su accionar, inciden en las estructuras de distribución social sobre los costos de producción, generan problemas de carácter social y ambiental debido a la entropía; $y$ para nuestros efectos, proveen condiciones de inclusión o de exclusión social, o más precisamente, pueden generar una integración social excluyente.

Esta discusión pone de manifiesto la necesidad de que analistas, investigadores, formuladores de política y tomadores de decisiones, tengan conciencia de la importancia que tiene la "justicia distributiva", que está implícita en sus praxis, referida a la relación entre CTI y desigualdad social (Cozzens, 2007). Además, deja claro que esta "justicia distributiva" debe llegar a transformarse en una serie de valores públicos (Bozeman y Sarewitz, 2005) con miras a consolidar una ciencia democrática, que tome en consideración los problemas $y$ las visiones de "los de abajo" (Woodhouse y Sarewitz, 2007) y donde la comunidad políticocientífica (Granados y Viales, 2013) (conjunto de actores políticos que formulan políticas científicas y tecnológicas, $y$ que toman decisiones en esta materia) considere el criterio de los científicos como expertos (Viales, Arellano y Granados, 2012), para construir políticas de alcance nacional, regional y local (Bhushan,
2015). Finalmente, es imprescindible la generación de mecanismos de rendición de cuentas por parte de los tomadores de decisiones (Biegelbauer y Hansen, 2011).

\section{LA RELACIÓN CIENCIA↔SOCIEDAD EN LA "SOCIEDAD RED”: ¿UNA RELACIÓN NUEVA?}

Si bien, la relación entre Ciencia y Sociedad es intrínseca, lo que sí ha cambiado es la historicidad de la relación, es decir, la manera en que se han vinculado ambos campos y la forma en que se ha interpretado esa relación, en distintas épocas y en espacios históricos diferentes.

En la actualidad, en el contexto de la globalización contemporánea, la convivencia entre ciencia y sociedad, que puede implicar relaciones de cooperación y de conflicto, es compleja. Por una parte, el paradigma tecnocientífico ha llevado a concebir a la ciencia y la tecnología como el núcleo de la economía y de la sociedad, de donde se desprende la visión de que los avances en estos ámbitos implican beneficios para la sociedad. Esta visión ha generado muchas expectativas sociales sobre el papel de la ciencia en la resolución de problemas fundamentales y cotidianos.

La denominada "sociedad de la información y del conocimiento", sobre todo las implicaciones éticas de los avances en CTI y tecnociencia, especialmente las percepciones de los riesgos concomitantes a estos avances, han generado una actitud escéptica, cuando no indiferente, con respecto al desarrollo tecnocientífico. Cuando se logra integrar de manera total la ciencia y la tecnología se está en presencia de la tecnociencia, la cual distingue entre la ciencia, que busca fines teoréticos y la tecnociencia, que persigue fines prácticos, de allî que, en criterio de Queraltó (2003), la "sociedad tecnológica" origina una nueva racionalidad, basada en los fines prácticos y que rompe con la racionalidad científica que se había heredado de la modernidad. Esta situación quedó bien planteada por parte de la CEC (2000) para el caso de los países desarrollados, donde:

$\diamond \quad$ El desarrollo tecnocientífico ha ampliado las posibilidades de transformación de la naturaleza por parte de la sociedad. 
$\diamond \quad$ El desarrollo social y económico ha planteado nuevas demandas para las agendas de investigación científica.

$\diamond \quad$ Los intereses del mercado han presionado por un mayor avance en el conocimiento.

$\diamond \quad$ El cambio tecnológico y el cambio social han demandado una reflexión sobre el cambio de los valores y los principios de la vida en sociedad.

$\diamond \quad$ Esta reflexión ha sido muy importante en grupos que están más allá del poder político convencional, es decir que se ubican en la sociedad civil, lo que ha generado una erosión en la autoridad política convencional en materia de la orientación de la agenda de investigación científica, que ha potenciado establecer nuevas vinculaciones entre las personas científicas, las autoridades políticas, los cuadros directivos de los ámbitos económico e industrial $y$, lo que viene a ser novedoso, una nueva relación con el público.

Todo lo anterior puede y debería sentar las bases para establecer un nuevo contrato entre la ciencia, las autoridades científicas, las universidades, las industrias, las empresas, los gobiernos y por otra parte, la sociedad, los grupos de presión y el público, la ciudadanía. Una llamada de atención apunta hacia la necesidad de que las políticas de CTI tomen en cuenta las demandas de la sociedad, para que tengan sentido para el público en general (CEC, 2000).

No se debe perder de vista que entre la tecnología, la tecnociencia y la sociedad, existen posibilidades de interacción múltiples. La introducción de tecnología per se no asegura la productividad, la innovación, el aprendizaje, la creatividad y el emprendedurismo. Esto debido a que la sociedad red y la sociedad tecnológica tienen diferentes manifestaciones de acuerdo a la cultura, las instituciones y la trayectoria histórica de cada sociedad (Castells, 2007). Lo anterior permite superar la visión del proceso de innovación como uno de carácter lineal, como se estudiará a continuación.

\section{LA PRODUCCIÓN DE NUEVOS CONOCIMIENTOS Y} LA INNOVACIÓN: ¿¿UN PROCESO LINEAL?

Aunque existe acuerdo para definir la innovación como un proceso de generación de nuevas tecnologías, es importante considerar que implica tanto la innovación de procesos como de productos, asimismo, no es el resultado de las acciones de un actor "en solitario", sino que más bien constituye un proceso en el que interactúan eventos concomitantes, actores diversos e instituciones, tanto en sus fundamentos técnicos como científicos y en sus conexiones físicas con otras partes del conjunto más complejo, que supone el sistema económico. El conocimiento no es creado en forma aislada $y$ las empresas innovan en tanto se vinculan con competidores, proveedores, clientes y una diversidad de instituciones (Mallo, 2008). En los países latinomericanos también innovan otros actores institucionales, fundamentalmente las universidades públicas.

Varios factores pueden potenciar la innovación, entre ellos la inversión en capital humano, el uso de las Tecnologías de la Información y la Comunicación (TIC) y las políticas públicas de apoyo a la innovación; la producción, la producción a escala, la gestión, el marketing $y$ los productos, los procesos, los bienes y los servicios, pero todos estos serán más o menos efectivos en función del contexto en que se desarrollen o en el que existan obstáculos o viabilidades para su desarrollo. Por esta razón, es importante tomar en cuenta que la innovación es un proceso que vincula la Investigación y el Desarrollo $(\mathrm{I}+\mathrm{D}+\mathrm{i})$, que debe transmitirse por medio de la educación y que estos pilares se deben potenciar por medio de políticas de ciencia y tecnología (Mateo, 2006).

El postulado de que la ciencia produce conocimiento, que de manera espontánea, puede ser usado por la sociedad y que la aplicación de este no depende de la participación ciudadana, debe modificarse. En la actualidad, no se puede negar que el conocimiento científico y el know-how tecnológico no es el resultado solamente de las acciones de las instituciones especializadas, antes bien, estos se producen en un amplio espectro de participación de organizaciones $y$ estructuras, de redes que involucran 
a los grupos de investigación y a los usuarios, tanto públicos como privados (CEC, 2000). Por lo tanto, es necesario concebir el proceso de innovación como una actividad relacional, que replantea su definición original hacia una concepción nueva que implica un proceso social de innovación.

Según Mario Albornoz, sobre el origen global del "modelo lineal de innovación", los

...historiadores suelen ser coincidentes en señalar que el campo de lo que hoy entendemos como políticas públicas de ciencia y tecnología se expandió en casi todo el mundo a partir de la segunda guerra mundial bajo la inspiración de lo que actualmente se conoce como "modelo lineal", cuyo énfasis está puesto en el financiamiento a la investigación básica como principio dinamizador del proceso creativo y de la transferencia de los conocimientos al entorno social. Aquel modelo era el corazón de la propuesta formulada por Vannevar Bush al Presidente de los Estados Unidos, en nombre de la comunidad científica de su país, para dar respuesta al problema de la utilización de ciencia como instrumento para el logro de objetivos nacionales estratégicos. Muchos intelectuales $y$ dirigentes políticos latinoamericanos estuvieron atentos a tal fenómeno. El resultado fue que desde la década de 1950 las políticas destinadas a fortalecer la investigación y la transferencia de tecnología formaron parte de las estrategias conducentes al desarrollo de la región (Albornoz, 2009, pp.65-66).

Si bien, existen muchas críticas al modelo lineal de innovación, interesa resaltar el peso que sobre esta tiene la incertidumbre, en un contexto en el cual interactúan la ciencia, la tecnología, las instituciones y los actores. Nathan Rosenberg ha planteado que la anticipación de futuros usos $y$ de mercados para algunas innovaciones ha sido un fracaso, ya que ingresan al mercado en un estado muy primitivo $y$ sus usos potenciales aparecen luego de un proceso de mejoras que amplía sus aplicaciones prácticas y de las invenciones complementarias (Rosenberg, 1994).

Es este fracaso el que ha estimulado a empresas como Intel, a integrar investigadores externos a la compañía, así como proveedores y consumidores que garantizan la utilidad de las innovaciones, a partir de un modelo de innovación abierta, como lo ha definido Chesbrough (2003), en oposición a la cerrada que implicaba el control de la empresa de todo el proceso de innovación, aunque se debe dejar claro que esta propuesta promueve la mercantilización del conocimiento. Por las razones anteriores, se debe analizar la relación entre Ciencia y Gobernanza.

\section{LA RELACIÓN CIENCIA-GOBERNANZA- INVESTIGACIÓN RESPONSABLE E INNOVACIÓN}

La noción de gobernanza se nutre de tres vertientes principales: la primera, que se convirtió en dominante en la década de 1980, se asocia con el desarrollo y la implementación de políticas públicas con una perspectiva desde arriba (top-down), donde priva el punto de vista del gobierno; la segunda, que se amplió en la década de 1990, plantea la posibilidad de establecer un nuevo tipo de gobierno, en el cual no se ejerza el poder de arriba hacia abajo, es decir de manera jerárquica, sino que más bien exista una mayor cooperación e interacción entre el Estado y los actores no estatales, al interior de redes de decisiones que vinculen lo público y lo privado; $y$ la tercera, que surge de manera casi paralela con la segunda, pone el énfasis en la necesidad de coordinar las acciones individuales, que se conciben como las originarias, para construir un orden social, un principio basado en el individualismo metodológico (Mayntz, 2005, pp.83-98).

Un nuevo contrato entre Ciencia y Sociedad, no puede dejar de lado la consideración de la gobernanza de la Ciencia, es decir, los procesos de interacción entre actores estratégicos, aquellos que cuentan con recursos de poder para influir en la toma de decisiones o en la solución de conflictos, y los actores subalternos, según las reglas del juego establecidas que se ejecutan a partir de instituciones formales e informales. 
Entonces la gobernanza es la "...interacción forjada por las reglas del juego" (Oriol, 2005, p.239) y precisamente esas reglas pueden transformarse en función de favorecer la innovación científica e institucional. En los países periféricos, subdesarrollados o pobres, esto plantea la necesidad de construir una nueva cultura política, de participación de la sociedad en la definición de la agenda de investigación en CTI. La cultura política se refiere a las culturas, creencias y valores que sustentan el sistema político y está cimentada en la institucionalidad que la valida, que puede estar constituida por instituciones formales (leyes, políticas públicas) y por instituciones informales (tradiciones, valores, identidad) (Turner, 2006, p.69).

En términos de la participación de actores estratégicos y de actores emergentes, la interacción debe integrar tanto a las autoridades políticas y científicas tradicionales como a la sociedad civil, o sea a las empresas, a los grupos organizados, a los gobiernos locales y a las minorías sociológicas. Estos actores, organizaciones $y$ redes, funcionan fuera del aparato formal (universidades, grupos de interés, ONG, "comunidades científicas", sindicatos, asociaciones profesionales, cámaras de comercio, asociaciones benéficas, asociaciones ambientalistas y ecologistas), por lo que se mueven en un espacio paralelo al Estado, pero aparte, por lo que intermedian las relaciones entre la ciudadanía, el Estado y el mercado.

La sociedad civil obtiene su legitimidad del fomento del interés público (Pope, 2000, pp.210-218) y este debe incorporar en su definición el fomento de la Ciencia. Esta es la "ciencia ciudadana”, es decir, el contrato entre no científicos y la investigación científica (Hollow, Roetman, Walter y Daniels, 2015), que implica una amplia gama de posibilidades y de resultados a partir de la participación pública.

Más recientemente, como plantean Arnaldi et ál. (2015), ha ganado espacio la noción de "responsabilidad" en la CTI, como un punto de encuentro entre estas actividades sustantivas y la sociedad, por lo que implica discusiones sobre la valoración de la tecnología, la ética y los riesgos de la ciencia y de la tecnología, así como la integración sociotécnica, que conlleva una "gobernabilidad responsable".

Este giro está influido por el "movimiento ascedente" que comprende la participación social como un mecanismo fundamental para incorporar el punto de vista de la sociedad tanto en el planteamiento de políticas científicas como en el proceso de toma de decisiones (Arnaldi et ál. 2015, p.82). Si estas condiciones no se cumplen, se podría estar en presencia de un déficit democrático en materia de políticas de CTI.

\section{LAS POLÍTICAS DE CIENCIA, TECNOLOGÍA E INNOVACIÓN ANTE LA AMPLIACIÓN DEL DÉFICIT DEMOCRÁTICO EN EL CONTEXTO DEL ESTADO NEOLIBERAL EN AMÉRICA LATINA}

Tal y como planteó Bijker hace una década, el valor de la ciencia es contextual (context specific) (Bijker, 2006, pp.109-125). En América Latina, hacia mediados del siglo Xx, se fomentó la participación estatal en la búsqueda de la autonomía tecnológica, lo que fue el corolario tecnocientífico del modelo de industrialización por sustitución de importaciones (ISI) (Bulmer-Thomas, 1998). De esta manera, el Estado se convirtió en un agente estratégico para la promoción de políticas públicas de Ciencia y Tecnología, que se construyeron con un sesgo antirural, bajo la lógica de que la brecha entre países pobres $y$ desarrollados se podía reducir mediante esfuerzos locales en el campo de la Ciencia y Tecnología.

La situación anterior varió hacia 1980, cuando el modelo neoliberal, con su lógica aperturista de integración competitiva al mercado global, valoró negativamente los esfuerzos anteriores, que fueron señalados como lentos, costosos e ineficaces, por lo que se promovió la asociación con capitales extranjeros, para promover una vía privilegiada de fomento de la Ciencia y Tecnología: la transferencia de tecnologías (Dagnino y Davyt, 2003, pp.97-138) que consolidó un patrón de transnacionalización y de privatización, que en algunos países desembocó en la maquila científica. Según Dagnino y Davyt (2003), los estados latinoamericanos, después de 1990, han restringido su función en 
materia de ciencia y tecnología. Los tres indicadores básicos que sustentan esta hipótesis:

1) No se crearon nuevas instituciones de ciencia y tecnología

2) Se estancaron los presupuestos nacionales de los sistemas de I+D.

3) Se han desestatizado algunas instituciones de I+D existentes.

En este contexto, en muchos países las empresas y no las universidades o los institutos públicos de investigación, se convirtieron en el nuevo "locus de la innovación", por lo que el mercado tendió a sustituir al Estado en materia de políticas de Ciencia y Tecnología, aunque no todos los países han recorrido ese camino. Se ha supuesto que la empresa va a invertir en la investigación universitaria, por lo que el Estado, a través de los gobiernos, no ha incrementado sus niveles de inversión para fomentar esta actividad, de ahí que las universidades han tenido que desarrollar políticas de investigación para no caer en las demandas y objetivos de corto plazo que, por lo general, orientan a las empresas (Dagnino y Davyt, 2003).

La falta de participación estatal en el fomento de la ciencia y la tecnología, ante el fracaso de la inversión de las empresas en esta materia, en diversos países, ha ampliado el déficit democrático que se señaló anteriormente, por lo que es importante considerar que este tipo de política no ha favorecido la cohesión social. Pero es válido cuestionarse ¿qué se podría hacer para superar este déficit democrático?

\section{LA RELACIÓN CIENCIA-SOCIEDAD: ¿CÓMO SUPERAR EL DÉFICIT DEMOCRÁTICO EN LA FORMULACIÓN DE POLÍTICAS PÚBLICAS DE CIENCIA, TECNOLOGÍA E INNOVACIÓN PARA LA COHESIÓN SOCIAL?}

Por lo general, en términos discursivos, las políticas científicas en América Latina identifican la producción de conocimiento como insumo para la resolución de problemas sociales. En gran parte, esta situación se explica por la persistencia de la retórica del modelo lineal de innovación; en el cual los procesos de innovación se sustentan en la investigación básica o fundamental, que pasan luego a la investigación aplicada y al desarrollo experimental, y finalmente se transforman en innovaciones que llegan al mercado (Kreimer y Zabala, 2006).

Sobre la base de esa idea, se creó la noción de la "relevancia social de los conocimientos científicos", pero ha resultado una idea que se ha construido desde arriba, por parte del Estado, del mercado y de las élites científicas, lo que deja al descubierto una contradicción: prácticamente, se plantea la existencia de un vínculo mágico entre la producción de conocimientos y la atención de problemas sociales (Kreimer y Zabala, 2006) por lo que los diferentes actores que participan del proceso son invisibilizados.

Así aparece el déficit democrático en la formulación de política públicas de CTI, que no se orienta a la concreción de buenas prácticas para lograr la cohesión social, puesto que:

...precisamente quienes están afectados por problemas sociales, que son indicados como las 'víctimas' de una situación que los perjudica, que los somete a una situación de riesgo, son al mismo tiempo aquellos que tiene más dificultades para generar una 'voz' para formular sus demandas en la esfera pública...(por lo que) la mayor parte de los problemas sociales que atañen a los sectores más carenciados de la sociedad, suelen ser formuladas por 'voceros' que gozan de legitimidad social para intervenir en la esfera pública (Kreimer 2007, p.4).

Por la razón anterior, se debe fomentar nuevas formas de diálogo y un enfoque participativo de políticas públicas (bottom-up), por lo que el elemento clave de democratización de las políticas de CTI, es fomentar la participación informada, para poder seleccionar entre las opciones posibles que ponen al alcance el desarrollo científico y tecnológico, de una manera responsable.

Las relaciones Estado/mercado/sociedad e individuo/comunidad/sociedad son fundamentales para comprender la objetivación y las subjetividades que subyacen en la problemática 
del desarrollo científico en América Latina, por lo que se debe tomar en cuenta:

...los actores se movilizan según sus preferencias e intereses. Y que pretenden influir, condicionar, bloquear o activar las decisiones públicas utilizando todo tipo de recursos...(Las decisiones de los actores públicos)...son interpretadas como decisiones de todos, y se puede argumentar que responden a los intereses generales, aunque lo cierto es que son casi siempre fruto de la interacción y negociación entre actores, $y$ de compromisos. Por tanto, son representativos de aquello que coyunturalmente se entiende como intereses generales (proceso en el que, en última instancia, priva el poder de persuasión, que implica la)...capacidad de definir conceptual y cognitivamente el problema a resolver (Subirats, 2001, p.260).

Por encima de los detalles técnicos, prevalece la posición de quienes son capaces de convencer a los otros actores: esos serían los actores estratégicos, que formarían parte de una élite; si se buscan grandes acuerdos, puede existir un ambiente más democrático, pero las reformas también pueden ejecutarse de manera más directiva, lo que depende del contexto de análisis. En este sentido, es vital la definición o formulación de un problema científico-social, para luego proceder con la formulación de las políticas públicas.

El déficit democrático, en términos de la formulación de políticas de CTI, se refiere al hecho de que los actores de la sociedad civil tienen oportunidades limitadas de impactar en la formulación de propuestas de política, y de hecho, muchos de ellos no aspiran a hacerlo. Por lo que en una sociedad, con disparidades o asimetrías, habría que evitar que la agenda de la política científica sea impulsada solo por parte de la "élite científica" (Viales, Arellano y Granados, 2012) y favorecer un papel creciente del desarrollo de iniciativas de la sociedad civil, donde puede incluirse a los científicos que están fuera de la élite y la concreción de "coaliciones promotoras".

Según Subirats, habría que trabajar en la dirección de solucionar las disparidades entre los actores, en términos de la desigualdad de recursos, por lo que él recomienda construir una red que garantice la: interdependencia entre actores; continuidad en su interacción; falta de autoridad "soberana"; capacidad de negociación constante y capacidad de encontrar objetivos, más o menos comunes con los que mantener la interacción. Esto se debe a que en la formulación de políticas públicas, es crucial que existan ideas de fondo, que son propuestas por comunidades epistémicas, que a la vez pueden tratar de imponer sus ideas sobre las de otras comunidades (Subirats, 2001, p.260).

La propuesta para favorecer la superación del déficit democrático reseñado, consiste en consolidar el diálogo entre las esferas que potencian la relación entre CTI y sociedad, que necesariamente debe involucrar al Estado, para canalizar los intereses y las demandas de la sociedad civil; de la ciencia y la investigación científica; de las empresas, las industrias y el mercado, así como de la tecnociencia, con esta relación se debe favorecer la innovación (Figura 1).

De allí que es necesario discutir cómo se construyen los problemas sociales y su relación con los tipos de conocimiento científico. 
FIGURA 1

EL DIÁLOGO ENTRE CIENCIA, TECNOLOGÍA, INNOVACIÓN Y SOCIEDAD PARA LA FORMULACIÓN DE POLÍTICAS PÚBLICAS DE CIENCIA PARA LA COHESIÓN SOCIAL EN PAÍSES PERIFÉRICOS

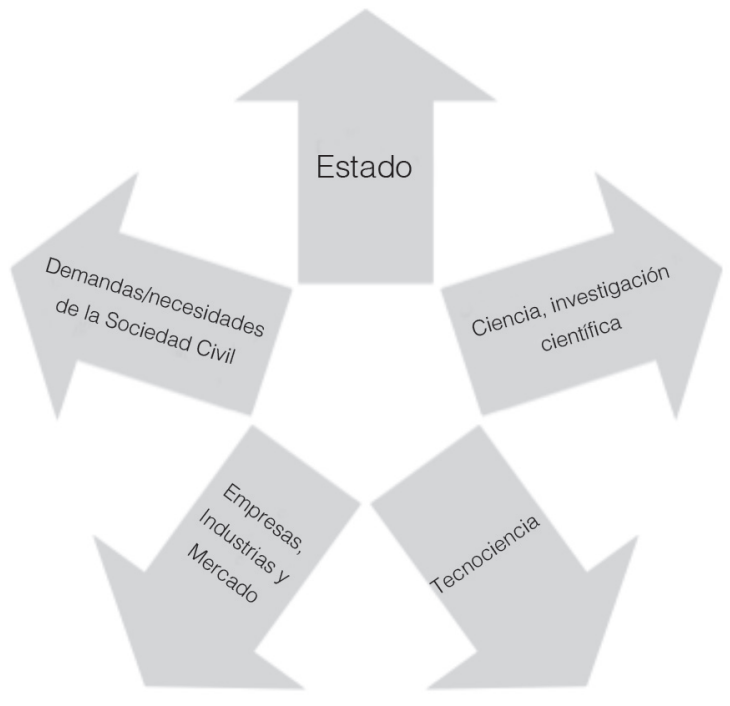

Fuente: Viales, 2010, p.21-40.

¿EN QUÉ FASE DE LA FORMULACIÓN DE UNA POLÍTICA CIENTÍFICA SE DEBE ABRIR EL ESPACIO PARTICIPATIVO PARA LA SOCIEDAD?

Las políticas públicas son "un conjunto encadenado de decisiones y de acciones resultado de las interacciones estructuradas y repetidas entre diferentes actores públicos y privados, que por diversas razones están implicados en el surgimiento, la formulación y la resolución de un problema políticamente definido como público" (Subirats et ál. 2008).
Como la construcción de políticas públicas es un proceso, lo primero que se debería hacer es partir del análisis del contexto existente, nacional, transnacional y global; abrir el proceso de formulación a la participación pública en la fase en que se percibe y se define el problema de política pública, por medio de una transformación de las reglas de juego (formales e informales) que han imperado en la formulación de políticas científicas para, finalmente, incidir sobre el contexto inicial y promover la generación de uno nuevo (Figura 2). 
FIGURA 2

ETAPAS DE LA POLÍTICA PÚBLICA: SUBIRATS. ¿QUIÉNES Y PARA QUIÉNES?
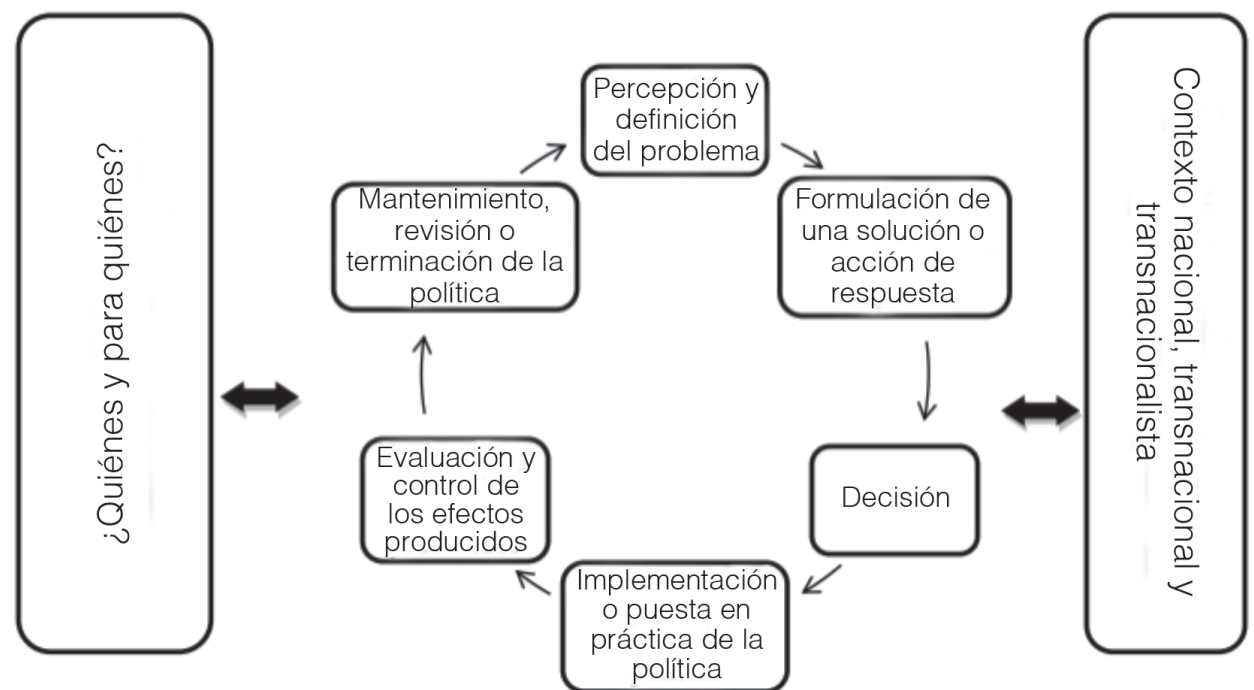

Fuente: $\quad$ Elaboración propia a partir de Subirats et ál, 2008.

Para el logro del objetivo anterior, se debe partir de una visión constructivista para la construcción social de los problemas de política pública. En ese sentido, es claro que no todos los problemas sociales se convierten, de manera objetiva, en problemas públicos. Garraud (1990), citado por Subirats et ál. (2008), propone tres condiciones para la construcción de un problema público, aunque es importante concebirlas de manera flexible: el surgimiento de una demanda por parte de grupos sociales determinados, que podría incluir comunidades científicas o grupos de investigación, según nuestro criterio; el desarrollo de un debate público, a partir de mecanismos participativos; y la existencia de un conflicto/controversia entre los grupos sociales organizados y la comunidad político-científica y las comunidades científicas (Figura 3).

Desde nuestra perspectiva, la formulación de políticas científicas debe contemplar la forma de integración social nacional y regional-local, sobre la base de una matriz de componentes, en función de una concepción $y$ de una representación de la propia cohesión social, a partir de una moral definida como general, que impone valores comunes que pueden ser construidos desde arriba $y / 0$ desde abajo; por ejemplo: participación democrática, respeto a la diversidad, a la dignidad, reconocer la diferencia, igualdad, acceso a oportunidades y recursos). 
FIGURA 3

TRES CONDICIONES PARA LA CONSTRUCCIÓN DE UN PROBLEMA PÚBLICO

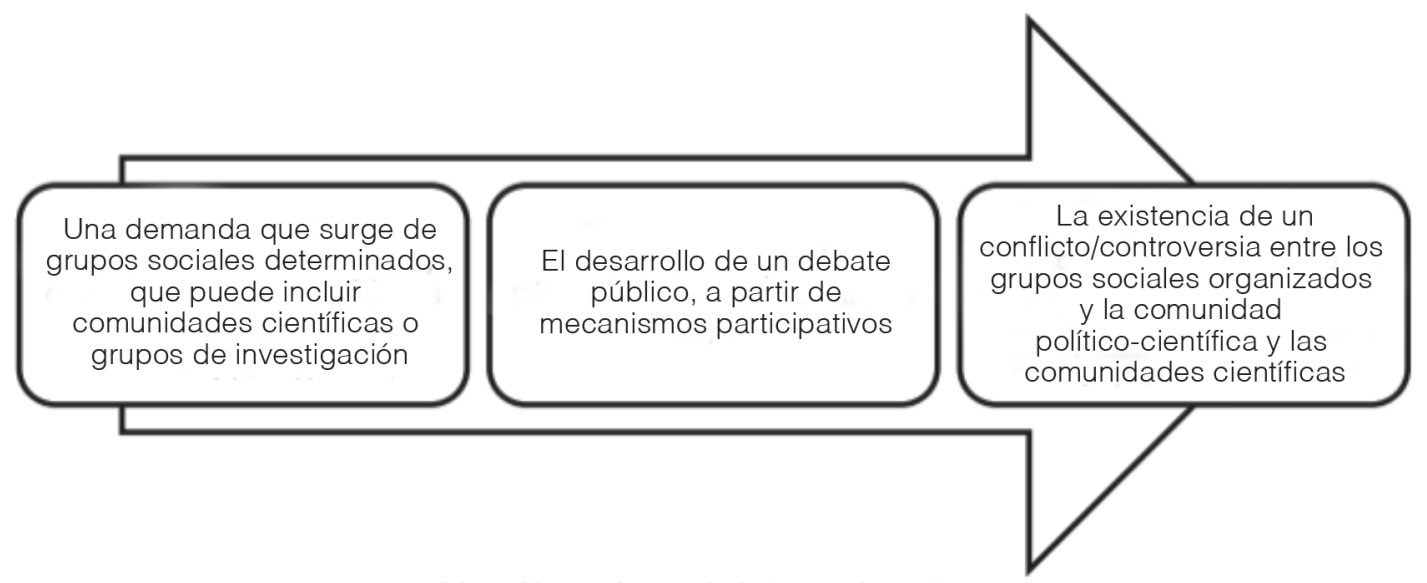

Fuente: Elaboración propia a partir de Garraud, 1990.

A esos componentes se debe agregar el del "desarrollo intercomunitario", que se oriente a promover "...una visión compartida de la sociedad tanto a nivel local como nacional" (Espinosa, 2010). Esa visión compartida, de manera ideal, debe partir del respeto "intranacional", en el sentido de conocer y garantizar el respeto de los regionalismos — si existenen función de la superación de las disparidades $y$ de las inequidades regionales, al interior de un país y también se puede incluir una visión transnacionalista, en el sentido de que se debe tomar en cuenta el contexto glocal de estas relaciones, sobre todo en el campo de la ciencia $y$ de los determinantes de la relación entre ciencia $y$ periferia.

En América Latina, el reformismo neoliberal, desde mediados de la década de 1980, ha generado políticas de CTI que apostaron por la creación de "sistemas nacionales de innovación”. Tal y como plantea Kreimer:

Se crearon diferentes instituciones 'de interfase' entre los institutos de investigación y desarrollo y las empresas productivas: parques y polos tecnológicos, incubadoras de empresas, secretarías de vinculación tecnológica en las universidades nacionales e institutos públicos de Investigación y Desarrollo (I+D). Complementariamente se crearon nuevos mecanismos de financiación - concebidos como un sistema de premios y castigos - con dos objetivos: inducir la reorientación de la investigación hacia la resolución de problemas tecnoproductivos y apoyar financieramente la innovación tecnoproductiva de las empresas locales... El aumento de la presión por producir conocimiento con valor de cambio fue internalizado por los productores de conocimientos, ya sea como retórica destinada a la mera legitimación o como un cambio sustantivo de las prácticas: la mayor parte del conocimiento producido es definida, por los propios investigadores, como 'aplicable' (Kreimer, 2010, p.49).

Pero muchas veces este tipo de desarrollos resultaron en Conocimiento Aplicable No Aplicado (CANA) (Kreimer y Thomas, 2005), por lo que en la formulación de políticas se deben contemplar los problemas sociales para convertirlos en Conocimiento Aplicable Sí Aplicado (CASA). 
PROBLEMAS SOCIALES Y TIPOS DE CONOCIMIENTO CIENTÍFICO: ¿DE QUIÉNES Y PARA QUIÉNES?

Hernán Thomas ha señalado que en la relación ciencia-cohesión social es básico articular las acciones de:

...al menos tres niveles de usuarios del conocimiento generado: actores institucionales vinculados al proceso de producción e implementación de Tecnologías Sociales, actores políticos vinculados a los procesos de policy making y toma de decisiones, actores comunitarios y usuarios finales de Tecnologías Sociales (Thomas, 2011).

Pablo Kreimer y Juan Pablo Zabala han dejado claro que tanto los problemas sociales como los conocimientos científicos se construyen mediante procesos en los que participan diferentes actores sociales y que, en los países periféricos, existe una escasa apropiación social del conocimiento científico en comparación con los países desarrollados, por lo que resulta fundamental dilucidar ¿cómo se construyen los conocimientos científicos? y ¿cómo estos son afectados (o deberían serlo) por la construcción de su utilidad social? (Kreimer y Zabala, 2006). Esta temática debe constituirse, como lo ha hecho en otros países latinoamericanos, sobre la base del enfoque CTS, en un campo de investigación.
Como puede observarse a partir de la Figura 4, desde nuestra perspectiva, los actores que deben participar en la formulación de políticas científicas para la cohesión social son diversos. La ventaja es que ahora se puede asumir que son diferentes, por lo que se potencia su participación pero es necesario que estos actores establezcan vínculos que se comuniquen a partir de diversos mecanismos en la búsqueda de acuerdos. Esta alternativa es valiosa, puesto que ha privado una falta de diálogo y la defensa de los cotos particulares e institucionales que rodean a cada actor particular.

En varios países, la crisis del modelo neoliberal ha permitido que se vuelva a visualizar al Estado (sector público) como el actor estratégico que puede guiar este nuevo diálogo. Este contrato entre Ciencia y Sociedad, como se planteó anteriormente, puede llevar a los demás actores a promover la innovación además de la participación ciudadana en la toma de decisiones en esta materia, lo cual facilitaría el desarrollo de una perspectiva de innovación social. Como mediadores entre los intereses del Estado, el mercado y la sociedad civil, se podría potenciar el papel de las universidades públicas y de las empresas, así como de los institutos públicos de investigación, pero se debe tomar en cuenta el surgimiento de intereses públicos no estatales, que han surgido precisamente por la erosión de la intervención estatal en algunos campos, que había sido promovida por el reformismo neoliberal. 
FIGURA 4

LOS ACTORES DE LAS POLÍTICAS PÚBLICAS EN CIENCIA, TECNOLOGÍA E INNOVACIÓN EN LA TRANSICIÓN A LA SOCIEDAD RED Y A LA SOCIEDAD TECNOLÓGICA DE LOS PAÍSES PERIFÉRICOS

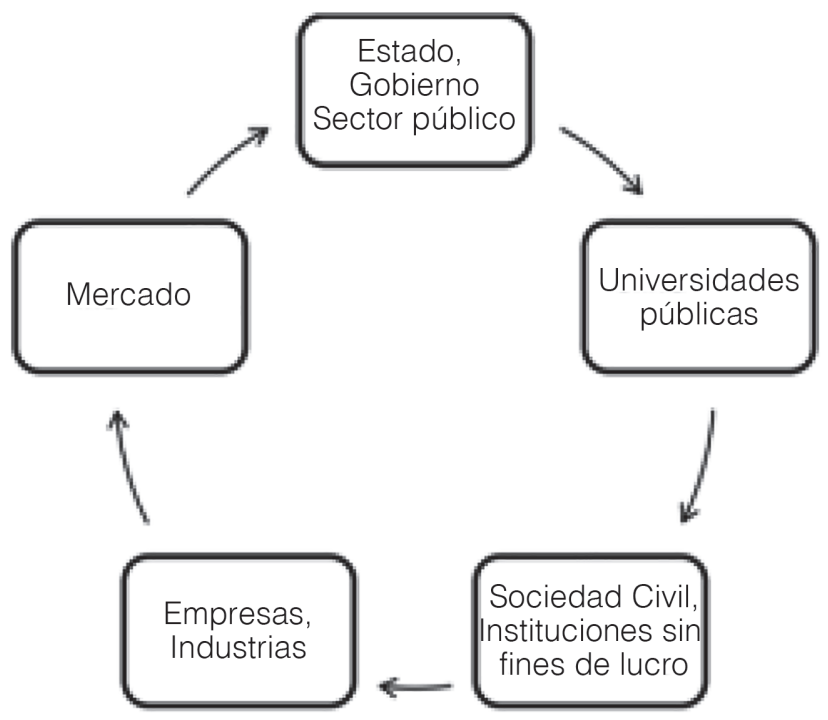

Fuente: Viales, 2010, p.21-40

Es importante considerar que no todo lo que es científicamente posible y tecnológicamente viable es deseable o admisible. Esta realidad no debe reñir con el principio de la libertad para investigar, que se puede comprender en dos sentidos: uno para construir y desarrollar la investigación, $y$ el otro de acceso al conocimiento. A esta discusión, se debe sumar la del secreto industrial y la patentabilidad del conocimiento (CEC, 2000).

En la actualidad, se abren nuevas perspectivas para la utilización de la "ciencia para la acción pública”, que pasan por la nueva relación entre ciencia y democracia para la formulación de políticas públicas, que tome en cuenta los impactos sociales de la ciencia, según ha planteado Santos (2004). Sobre la base de este planteamiento, se esbozar algunos fundamentos básicos para la formulación de políticas participativas para la cohesión social.
FUNDAMENTOS BÁSICOS PARA ABRIR LA DELIBERACIÓN EN LA FORMULACIÓN DE POLÍTICAS DE CTI

Se debe pasar del conocimiento relevante al conocimiento socialmente sólido, como ha planteado Nowotny, puesto que la:

...sociedad espera cada vez más las contribuciones de la ciencia, lo que implica una mayor integración de grandes dimensiones sociales al trabajo de los científicos. Estas pueden ser consideraciones éticas o ambientales, o pueden estar relacionadas con futuros usos específicos del conocimiento, incluso en la investigación básica...La participación, especialmente la que procede de la base, crea un sentido de propiedad y permite que surja una visión de ciudadanos científicos (2012, p.340). 
La participación-deliberación de la ciudadanía, por medio de un proceso participativo, puede tener diferentes grados que se materializan en mecanismos como: la información, la comunicación, la consulta, la deliberación y hasta la participación en la toma de decisiones; si bien cada grado es incremental, esto no significa que el de la decisión es más democrático que los demás, porque eso dependerá del contexto en que se lleve a cabo la participación.
El tema fundamental es fomentar la participación y la incidencia de la sociedad civil en las políticas científicas, que las pueda dotar de un carácter democrático y más igualitario $(\mathrm{Pa}-$ gano, Costas y Sverdlick, 2007). La efectividad de estos fundamentos dependerá de las estructuras y de los mecanismos de participación que se creen, del origen, del grado de incidencia y de la relación posible entre demandas y políticas públicas de ciencia (Figura 5).

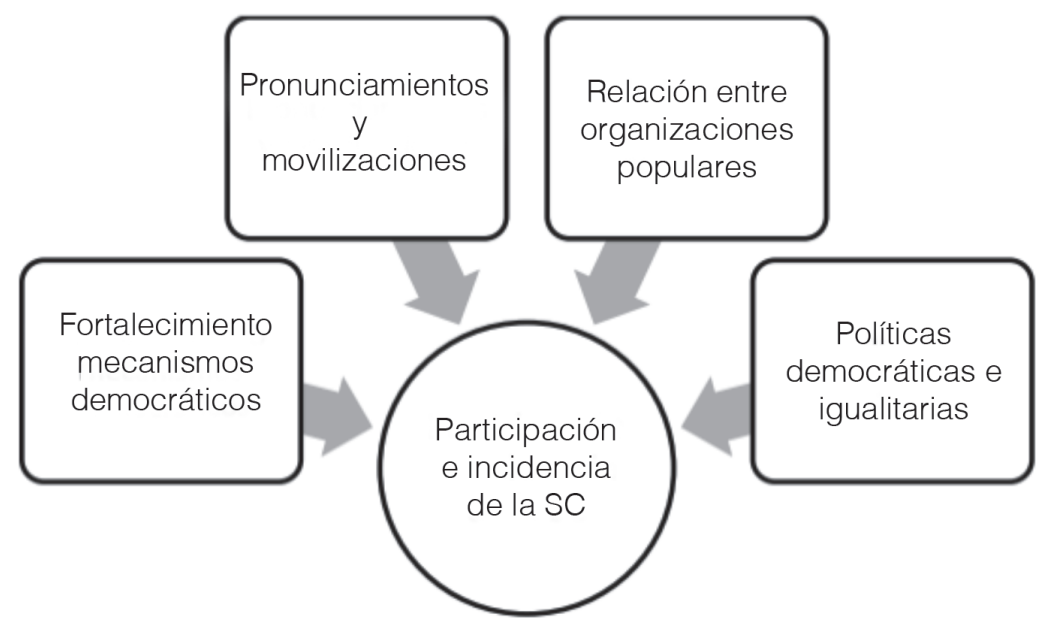

Fuente: Adaptación a partir de Pagano, Costas y Sverdlick, 2007

Estos elementos llevarán a un proceso de empoderamiento individual y social (Kaufman, 1997), con miras a la búsqueda de desarrollo socioeconómico y de tranformación política que en términos de la cohesión social, se deben ampliar en el sentido de incluir el desarrollo científico y socio-técnico como un elemento básico de integración social (Figura 6). El community power ("poder comunal") potencia y demanda la organización de la participación comunitaria de base; la generación de mecanismos participativos para la toma de decisiones; el surgimiento de nuevas estructuras administrativas adaptadas a la nueva realidad; la formulación de planes nacionales de desarrollo científico y tecnológico, así como de innovación, que respondan a los problemas de una comunidad, de un país o de una región, detectados y construidos por los propios actores y no "desde arriba". Asimismo, permite pensar en la posibilidad de trabajar en la construcción de políticas públicas de cohesión social regional (Viales, 2014). 
FIGURA 6

LAS BASES DEL COMMUNITY POWER Y LA FORMULACIÓN DE POLÍTICAS CIENTÍFICAS

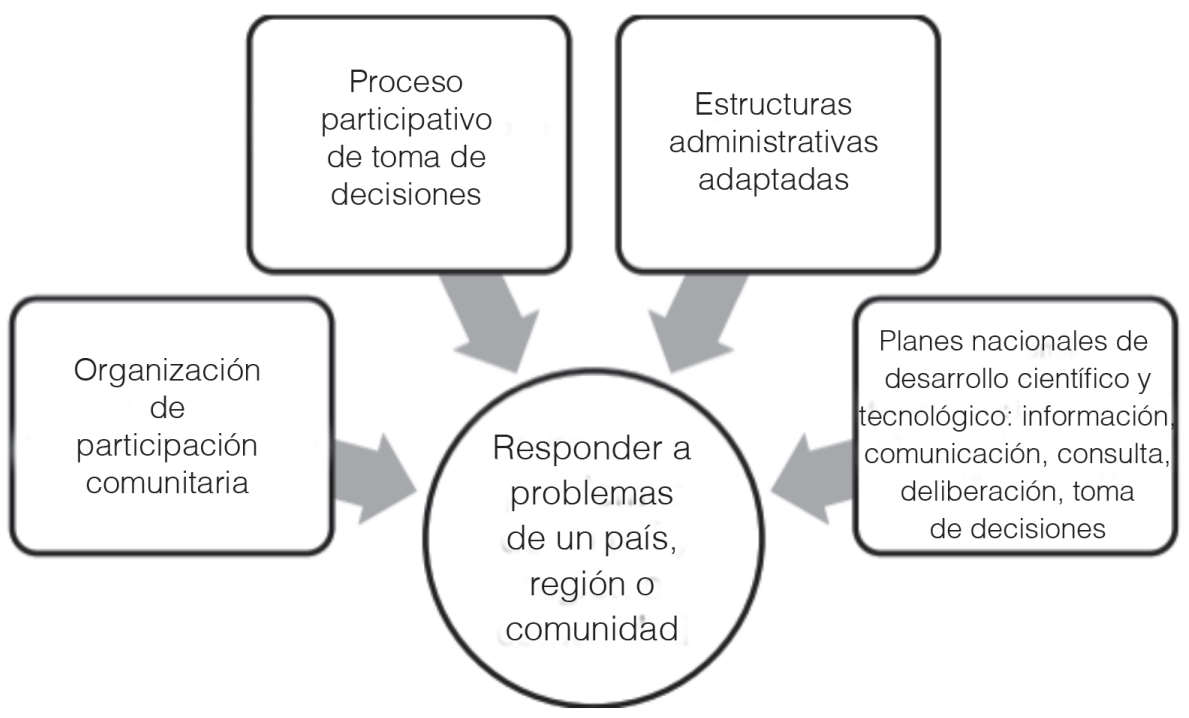

Fuente: $\quad$ Elaboración propia y adaptación a partir de Kaufman, 1997.

Este planteamiento se debe complementar con el desarrollo de una ciudadanía sociotécnica, que Thomas (2011) sugirió y que Tula (2011) bosquejó como “...la propia inclusión crítica de los usuarios-beneficiarios en las decisiones sobre el diseño, producción e implementación de los sistemas tecnológicos" (p.172) y de las políticas científicas. Siguiendo el plantamiento de Arditi (2004), la ciudadanía sociotécnica sería un cuarto tipo de ciudadanía (Figura 7) que coexiste con la primaria, centrada en la democracia representativa; con la secundaria, que ha crecido a partir del resurgimiento de la sociedad civil y con la terciaria, que ha ampliado la partipación en el ámbito supranacional.

Construir mecanismos participativos para generar espacios para la formación de la ciudadanía sociotécnica constituye un campo de debate y de acción importante que deben abordar los estudios CTS en América Latina, pero tomando en cuenta su contexto institucional y de desarrollo de la sociedad civil de cada país. 
FIGURA 7

COEXISTENCIA DE CUATRO TIPOS DE CIUDADANÍA: INCLUSIÓN DE LA CIUDADANÍA SOCIO-TÉCNICA

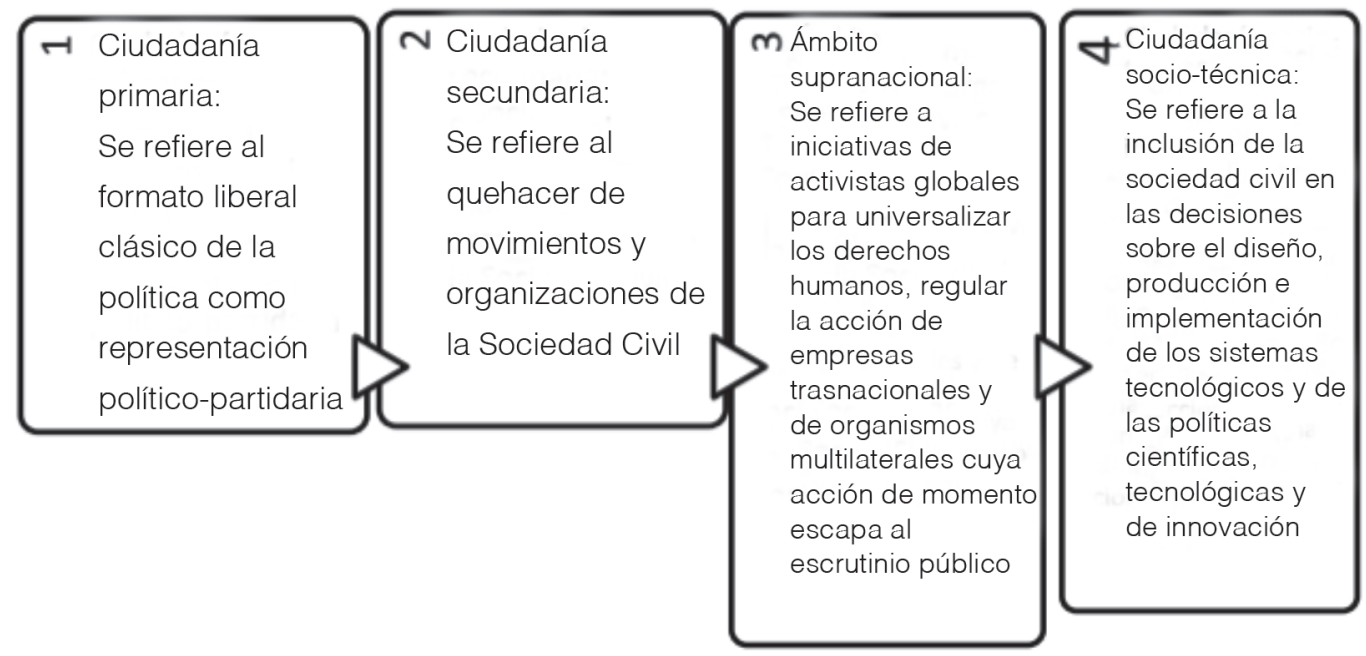

Fuente: Elaboración propia a partir de Arditi (2004) y de Thomas (2011).

\section{CONCLUSIÓN: HACIA LA FORMULACIÓN DE POLÍTICAS DE CTI PARA LA COHESIÓN SOCIAL}

Desde la perspectiva expuesta, es importante generar políticas de CTI y tecnociencia para la cohesión social, por lo que se debe superar la brecha existente entre ciencia y sociedad a partir del establecimiento de un nuevo contrato o pacto que supere la distancia entre estos ámbitos y la participación ciudadana.

Por esta razón, además de comunicar los hallazgos científicos y los avances en la investigación, las personas ciudadanas deben contar con una cultura científica, técnica y sociotécnica básica, que se puede definir como un conjunto de conocimientos y competencias que se adquieren al final de ciclo escolar o profesional obligatorio, pero que puede incrementarse por medio de la educación continua y el acceso a las TIC.

Lo anterior generaría una ciudadanía sociotécnica que podría permitir una mayor interacción entre los actores involucrados en los procesos científicos y a la vez, los científicos podrían tomar conciencia de la importancia de que los impactos sociales de la ciencia se contemplen como un elemento fundamental de todo proyecto de investigación.

Es necesaria la construcción de mecanismos participativos $y$ deliberativos para lograr esta coordinación, donde el "poder comunal" juega un papel fundamental. Asimismo, se debe reconocer la diversidad de actores que participan del proceso de investigación científica y de innovación, con el fin de superar el déficit democrático en la formulación de políticas científicas.

Será fundamental que se reconozca el papel de la CTI como agentes de cambio social $y$ cuyos resultados juegan un papel de primer orden en la integración social incluyente, que permite superar brechas sociales para concretar una nueva cohesión social en nuestros países latinoamericanos. 
BIBLIOGRAFÍA

LIBROS

Bijker, W. (2006). Science and technology policies through policy. En: Box L. y Engelhard, R. (ed.) Science and Technology Policy for Development, Dialogues at the Interface. London: Anthem Press.

Bulmer-Thomas, V. (1998). La historia económica de América Latina desde la Independencia. México: Fondo de Cultura Económica.

Castells, M. (2005). The Network Society: From Knowledge to Policy. En: Castells, M. y Cardoso, G. (Eds.). The Network Society: From Knowledge to Policy. Washington: Johns Hopkins Center for Transatlantic Relations, 3-21.

Comisión Económica para América Latina $y$ el Caribe-CEPAL. (2007). Cohesión social. Inclusión y sentido de pertenencia en América Latina y el Caribe. Santiago de Chile.

Comisión de las Comunidades Europeas. (2000). Science, society and the citizen in Europe SEC. Brusels: The Commission.

Dagnino, RTH. $y$ Davyt, A. (2003). El pensamiento en Ciencia, Tecnología y Sociedad en Latinoamérica: una interpretación política de su trayectoria. En: RTH Dagnino, RTH. y Davyt, A. (Eds.). Ciencia, Tecnologia e Sociedade. Uma reflexão latinoamericana: um tributo a Amilcar Herrera. San Pablo: Cabral-oEI, 97-138.

Espinosa, N. (2010). Convivencia Democrática e Inclusión Social. Una Aproximación desde el Liderazgo. En: Espinosa, N. Documentos. Gobernabilidad y Convivencia Democrática en América Latina. San José: FLACSO/Secretaría.

Farrell, G. (2010). Cohesión social, gobernabilidad y convivencia democrática: el enfoque propuesto por el Consejo de Europa. San José, FLACso/ Secretaría General.

Gibbons, M., Limoges, C., Nowotny, H., Schwartzman, S., Scott, P. y Trow,
M. (1994). The New Production of Knowledge. The dynamics of science and research in contemporary societies. Londres: Sage Publications.

Kaufman, M. (1997). Community Power, Grassroots Democracy, and the Transformation of Social Life. En: Community Power and Grassroots Democracy. The transformation of Social Life. Ottawa: Zed Books.

Kreimer, P. (2007). Conocimiento científico y problemas sociales: ¿quién construye a quién? Una discusión general y un ejemplo particular: la enfermedad de Chagas. En: Sarti, I. (Ed.). Ciencia, Politica e Sociedade. As Ciencias Sociais na América do Sul. Porto Alegre: Universidade Federal do Rio Grande do Sul.

Kreimer, P. (2010). Ciencia y Periferia. Nacimiento, muerte y resurección de la Biología Molecular en la Argentina. Buenos Aires, EUDEBA.

Kreimer, P. y Thomas, H. (2005). What is CANA-AKNA? Social Utility of Scientific and Technological Knowledge: challenges for Latin American Research Centers. En: Development through knowledge? A new look at the global knowledge-based economy and society. Ginebra: IUED.

Mayntz, R. (2005). Nuevos desafíos de la teoría de la gobernanza. En: Cerrillo, A. (Ed.). La gobernanza hoy: 10 textos de referencia. Madrid, Instituto Nacional de Administración Pública, 83-98.

Pagano, A., Costas, P. y Sverdlick, I. (2007). Participación e incidencia de la sociedad civil en las politicas educativas. Buenos Aires: Fundación Laboratorio de Políticas Públicas.

Queraltó, R. (2003). Ética, tecnología y valores en la sociedad global. El caballo de Troya al revés. Madrid: Tecnos.

Rosenberg, N. (1994). Exploring the Black Box. Technology, Economics and History. Cambridge: Cambridge University Press.

Sismondo, S. (2010). An Introduction to Science and Technology Studies. WileyChichester U.K.: Blackwell. 
Subirats, J., Knoepfel, P., Larrue, C. y Varone, F. (2008). Análisis y gestión de políticas públicas. Barcelona: Ariel.

Szmukler, A. (2008). Culturas de desigualdad, Democracia y Cohesión Social en la Región Andina. San Pablo, Brasil y Santiago de Chile: Instituto Fernando Henrique Cardoso y Corporación de Estudios para Latinoamérica.

Turner, B. (2006). Civic Culture. En: Turner, B. (Ed.). The Cambridge Dictionary of Sociology. Cambridge: Cambridge Univesity Press.

Viales, R. (2010). Las relaciones entre Ciencia, Tecnología, Tecnociencia, Innovación y Sociedad. Elementos para la formulación de políticas científicas para la cohesión social. En: Viales, R. (Ed.). El contexto, los problemas y los actores de la definición de politicas científicas para la cohesión social en América Latina: una visión desde Costa Rica. San José: Sociedad Editora Alquimia 2000, 21-40.

Viales, R. (2014). Hacia una propuesta de modelo de cohesión social regionallocal para Puntarenas en la actualidad, a partir de la incidencia de la Sede del Pacífico de la Universidad de Costa Rica. En: Bartels, J., Chavarría, B., Marín, JJ. y Viales, R. (Eds.). La minería en Bellavista-Miramar, Costa Rica: ¿Dónde quedó la riqueza? Historia, conflicto $y$ percepciones de una explotación. 18212012. San José: Nuevas Perspectivas/ Sede del Pacífico/Universidad de Costa Rica,155-168.

\section{PUBLICACIONES PERIÓDICAS}

Albornoz, M. (2009). Desarrollo y políticas públicas en ciencia y tecnología en América Latina. Revista de Investigaciones Politicas y Sociológicas, 8, (1), 65-75.

Arditi, B. (2004). Trayectoria y potencial político de la idea de sociedad civil. Revista Mexicana de Sociología, (1), 1-21.

Arnaldi, S., Quaglio, G., Ladikas, M., O’Kaine, H., Kharapiperis, T., Ravi, K. y Zhao, Y. (2015). Responsible governance in science and technology policy: Reflections from Europe, China and India. Technology in Society, (42), 81-92.

Bhushan, B. (2015). Perspective: Science and technology policy-What is at stake and why should scientists participate? Science and Public Policy, (42), 887-900.

Biegelbauer, P. y Hansen, J. (2011). Democratic theory and citizen participation: democracy models in the evaluation of public participation in science and technology. Science and Public Policy, 38, (8), 5889-597.

Bozeman, B. y Sarewitz, D. (2005). Public values and public failure in us science policy. Science and Public Policy, 32, (2), 119-136.

Casas, R. (2004). Las nuevas formas de producción de conocimiento: reflexiones en torno a la interdisciplina en las Ciencias Sociales. OMNIA, 20, (Especial), 263-274.

Casas, R. (2012). Social cohesion in distributive policies and the role of knowledge. Science and Public Policy, (39), 562-569.

Castells, M. (2007). Comnunication, Power and Counter-power in the Network Society. International Journal of Communication, 238-266.

Chesbrough, HW. 2003. The Era of Open Innovation. MIT Sloan Management Review, 44, (3), 35-41.

Cozzens, S. (2007). Distributive justice in science and technology policy. Science and Public Policy, 2, (34), 85-94.

Garraud, P, (1990). Politiques nationales: élaboration de l'agenda. L'Année Sociologique, (40), 17-41.

Harmon, S., Laurie, G. y Haddow, G. (2013). Governing risk, engaging publics and engendering trust: New horizons for law and social science? Science and Public Policy, (40), 25-33.

Hollow, B., Roetman, P., Walter, M. y Daniels, C. (2015). Citizen science for policy development: The case of koala management in South Australia. Environmental Science \& Policy, (47), 126-136. 
Jasanoff, S. (2003). Technologies of Humility: citizen particippation in governing Science. Minerva, (41), 223-244.

Granados, R.E. y Viales, R. (2013). La construcción del ofidismo como problema científico-social en Costa Rica: una trayectoria histórica y relacional (1881-1988). En: Arellano A., Chauvet, M. y Viales, R. (Eds.). Redes y estilos de investigación. Ciencia, Tecnología, Innovación y Sociedad en México y Costa Rica. México: Editorial Miguel Ángel Porrúa, 115-134.

Kreimer, P. (2006). ¿Dependientes o integrados? La ciencia latinoamericana y la nueva división internacional del trabajo. Nómadas, (24), 199-212.

Kreimer, P. y Zabala, JP. (2006). ¿Qué conocimiento y para quién? Problemas sociales, producción y uso social de conocimientos científicos sobre la enfermedad de Chagas en Argentina. Redes, 12, (23), 49-78.

Mallo, E. (2008). Innovación tecnológica $y$ sistemas de innovación. Una aproximación conceptual. Hologramática, (9), 9-121.

Mateo, J.L. (2006). Sociedad del Conocimiento. ARBOR, CLXXII, (718), 145-151.

Oriol, J. (2005). El concepto y el análisis de la gobernabilidad. Revista Instituciones $y$ Desarrollo, 14, (1), 239-269.

Pope, J. (2000). La Sociedad Civil. En: Pope, J. (Ed.). Libro de Consulta de Transparencia Internacional. México: Transparencia Mexicana, 210-218.

Santos, T. (2004). Politiques scientifiques, démocratie et mutations des institutions de savoir. RISS, (180), 275-286.

Subirats, J. (2001). El análisis de las políticas públicas. Gac Sanit, 15, (3), 259-264.
Thomas, H. (2011). Tecnologías sociales y ciudadanía socio-técnica. Notas para la construcción de la matriz material de un futuro viable. Ciência \& Tecnologia Social, 1, (1), 1-22.

Tula, F. (2011). Consumo tecnológico y educación tecnológica: fundamentos filosóficos para un proyecto futuro. Sociologias, (26), 154-175.

Vessuri, H. (2003). Science, politics, and democratic participation in policymaking: a Latin American view. Technology in Society, (25), 263-273.

Viales, R., Arellano, A. y Granados, R.E. (2012). Perceptions about the political-scientific community and its role in formulating the problems of public policy for science, technology and innovation in Costa Rica. Science and Public Policy, 39, (5), 613-617.

Wetmore, J. (2007). Introduction to special issue on science,policy and social inequity. Science and Public Policy, 34, (2), 83-84.

Woodhouse, E. y Sarewitz, D. (2007). Science policies for reducing societal inequities. Science and Public Policy, 34, (3) 139-150.

OTROS

Nowotny, H. (2012). ¿Salirse de la ciencia es salir de sincronía? En: UNESCO (Ed.). Informe sobre las Ciencias Sociales en el Mundo. 2010. Las brechas del conocimiento. México: UNESCO/Foro Consultivo, 337-340.

Uso de la Ciencia para la Cohesión SocialUCICOS. (2008). redIberoamericana sobre el uso del conocimiento científico para la cohesión social, CYTED, 608RT0349.

Fecha de ingreso: 11/07/2016 Fecha de aprobación:16/08/2016 